\title{
Downregulation of carbonic anhydrase IV contributes to promotion of cell proliferation and is associated with poor prognosis in non-small cell lung cancer
}

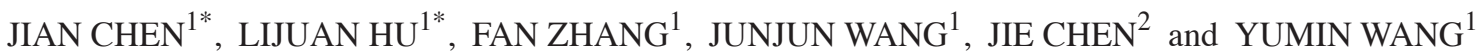 \\ Departments of ${ }^{1}$ Laboratory Medicine, and ${ }^{2}$ Intensive Care Unit, The First Affiliated Hospital \\ of Wenzhou Medical University, Wenzhou, Zhejiang 325000, P.R. China
}

Received March 24, 2017; Accepted August 4, 2017

DOI: $10.3892 / \mathrm{ol} .2017 .6740$

\begin{abstract}
The present study aimed to unveil the biological role of carbonic anhydrase IV (CA IV) and its association with clinical pathological characteristics and prognostic significance in non-small cell lung cancer (NSCLC). The relative expression level of CA IV was measured by reverse transcription-quantitative polymerase chain reaction in 114 resected NSCLC tumors and matched adjacent normal tissues and NSCLC cell lines. Overexpression and cell proliferation were assessed in human NSCLC cell lines transfected with CA IV mRNA by lentivirus-mediated technology. The association of CA IV expression with clinical pathological features and overall survival in 114 cases of NSCLC patients was analyzed. It was demonstrated that CA IV expression was significantly downregulated in NSCLC tumors and six cell lines. Reduced expression of CA IV was significantly correlated with lymph node metastasis. The overall survival of NSCLC patients with low CA IV expression was significantly shorter compared with the high expression group. Overexpression of CA IV suppressed cell proliferation in A549 and NCI-H1299 cells. The results indicate that low expression of CA IV promotes cell proliferation and serves as an indicator for poor prognosis in NSCLC.
\end{abstract}

\section{Introduction}

Lung cancer is the most common incident cancer and the leading cause of cancer death $(1,2)$. In 2015 , and approximately 733,300

Correspondence to: Dr Jie Chen, Department of Intensive Care Unit, The First Affiliated Hospital of Wenzhou Medical University, Fuxue Xian 2, Wenzhou, Zhejiang 325000, P.R. China

E-mail: chenjie991300@163.com

Dr Yumin Wang, Department of Laboratory Medicine, The First Affiliated Hospital of Wenzhou Medical University, Fuxue Xian 2, Wenzhou, Zhejiang 325000, P.R. China

E-mail: wym0577@163.com

*Contributed equally

Key words: non-small cell lung cancer, carbonic anhydrase IV, proliferation, prognosis new lung cancer cases are diagnosed and 610,200 patients died of lung cancer in China1. Non-small cell lung cancer (NSCLC) accounts for approximately $87 \%$ of lung cancer cases (3). Although the mainstays of treatment for lung cancer (such as surgery, radiotherapy, chemotherapy and targeted therapy) have made considerable progress, NSCLC remains an aggressive lung cancer associated with a poor prognosis. Long-term survival of lung cancer is less than $10 \%$ (4-6). Thus, finding new biomarkers for predicting progression and prognosis of NSCLC is warranted and urgently needed to improve clinical management of patients with NSCLC.

Carbonic anhydrase IV is one of twelve active human isozymes and one of four expressed on the extracellular surfaces of certain endothelial and epithelial cells, which catalyzes the reversible hydration of $\mathrm{CO}_{2}$ to $\mathrm{HCO}_{3}-$ and $\mathrm{H}+(7,8)$. Carbonic anhydrase IV (CA IV) was found in human normal tissues like kidney and lung, with the remarkable diversity in tissue distribution, subcellular location, and biological function $(9,10)$. Several CAs are reportedly involved in NSCLC tumorigenesis, progression, regulation of cell proliferation, target therapy and prognosis, excepted CA IV. For example, CA IX, CAXII, and CA I are upregulated in NSCLC tumor tissues and appear to act as oncogenes or prognostic factors (11-14). CA-RP VIII and CA IX expression in NSCLC are related to NSCLC cell invasion and proliferation $(15,16)$. CA IX serves as a target for anticancer therapy (17). A new study found that CA IV is frequently silenced in colorectal cancer and the silencing of CA IV is regulated by promoter hyper-methylation (18). Moreover, CA IV is a novel tumor suppressor in CRC which was found to be associated with the inhibition of the Wnt/ $\beta$-catenin signalling pathway. The normal lung expression of CA IV was found to be developmentally regulated in the luminal side of the alveolar capillary endothelium cells. We hypothesize that, like many other CAs, CA IV might be associated with NSCLC. Our preliminary studies using high-throughput microarrays and quantitative real-time RT-PCR (RT-qPCR) revealed that the expression of CA IV was downregulated in NSCLC tissues (1). However, the mechanism involved in the influences of CA IV on biological functions in NSCLC is very complicated and the exact clinical roles of CA IV are still remained unclear, making further validation is necessary. 
In the present study, the relative expression of CA IV is estimated by RT-qPCR in 114 resected NSCLC tissues compared to levels in their paired NT. The relationship between expression levels of CA IV, clinical pathological features and overall survivals in NSCLC patients is investigated. We overexpress CA IV mRNA basing on NSCLC A549 and NCI-H1299 cell lines by lentivirus-mediated technology.

\section{Materials and methods}

Patient samples. The 114 NSCLC tissues and corresponding adjacent normal tissues (NT) were obtained from patients who underwent surgery at the First Affiliated Hospital of Wenzhou Medical University, China, from August 2013 to October 2015. This study was approved by the Ethical Committee of the First Affiliated Hospital of Wenzhou Medical University, and all patients signed informed consent for the collection and use of their tissues for this study. The clinical pathological features of patients (Table I) were assessed according to the World Health Organization classification (19) and the TNM staging system. The NSCLC and matched NT samples were snap-frozen in liquid nitrogen immediately after resection. Patients were regularly followed up by telephone or other means, the longest follow-up time was 31 months. To investigate the association of CA IV expression with prognosis, the survival data was divided into high and low groups, a value superior or equal to 2 was defined as CA IV overexpression, based on the $2^{-\Delta \Delta \mathrm{Cq}}$ method (20).

Quantitative PCR. Approximately $100 \mathrm{mg}$ tissues from liquid nitrogen were cut off with a high-pressure sterile surgical scissors and then placed in a $4 \mathrm{ml}$ centrifuge tube treated with $0.1 \%$ DEPC water. Take $1 \mathrm{ml}$ of TRIzol reagent (Invitrogen, Carlsbad, CA, USA) into the centrifuge tube, then extracted the total RNA follow the reagent instructions. cDNA was reverse-transcribed from total RNA samples using an RT Reagent kit (Takara Bio, Dalian, China), based on the manufacturer's instructions. The measurement of CA IV and $\beta$-actin mRNA was performed by RT-qPCR with SYBR Premix Ex Taq in ABI 7000 instrument. CA IV forward primer: 5'-CTGGTGCTACGAGGTTCAA-3' and reverse primer: 5'-GCCTTGGTGGTGACGAT-3'; $\beta$-actin sense primer: 5'-CCTGGCACCC AGCACAAT-3', antisense primer: 5'-GCTGATCCACATCTGCTGGAA-3'. $2 \mu \mathrm{g}$ of total RNA were transcribed into cDNA and $\mathrm{PCR}$ reaction was performed in a final volume of $20 \mu \mathrm{l}$, containing $10 \mu \mathrm{l}$ of SYBR Premix (2x), $2 \mu 1$ of cDNA template, $1 \mu 1$ of each primer $(10 \mathrm{mM})$, and $6 \mu \mathrm{l}$ of double-distilled water. The quantitative real-time PCR reaction consisted of an initial denaturation step of $10 \mathrm{~min}$ at $95^{\circ} \mathrm{C}, 40$ cycles of $5 \mathrm{sec}$ at $95^{\circ} \mathrm{C}, 30 \mathrm{sec}$ at $60^{\circ} \mathrm{C}$, and a final extension step of $5 \mathrm{~min}$ at $72^{\circ} \mathrm{C}$. Each sample was performed in triplicate and the median was used to calculate the relative concentrations with $\beta$-actin as an internal control gene $(\Delta \mathrm{Ct}=\mathrm{Ct}$ median CA IV-Ct median $\beta$-actin), and $2^{-\Delta \Delta C q}$ in expression was calculated (20).

Cell culture. Six human NSCLC cell lines (SPCA-1, NCI-H1975, LTEP-a2, NCI-H1299, NCI-H441 and A549) and normal human bronchial epithelial BEAS-2B were all purchased from the Cell Bank of the Chinese Academy of Sciences and maintained with complete medium (containing $10 \%$ fetal bovine serum and $90 \%$ RPMI 1640 ) at $37^{\circ} \mathrm{C}, 5 \% \mathrm{CO}_{2}$, complete medium was changed at least once every two days.

Lentivirus-mediated overexpression vector transfection. A549 and NCI-H1299 cells were transfected overexpression vector targeting CA IV as well as a negative control (Genechem, Co., Ltd., Shanghai, China). Transfection was accomplished by seeding $2 \times 10^{5}$ cells into a six-well plate, and after $24 \mathrm{~h}$, the medium was aspirated and incubated with transfection complex, according to the manufacturer's protocols. The A549 and NCI-H1299 cells were infected with lentivirus for $72 \mathrm{~h}$ and the overexpression efficiency was detected by RT-qPCR.

Cell proliferation assay. Cell viability was evaluated by Cell Counting Kit-8 (Corning Inc, Acton, MA, USA) abiding by the manufacturer's protocols. Briefly, 3,000 cells of Stable transduced A549 and NCI-H1299 were suspended and seeded into a 96-well plate with supplemented medium (10\% fetal bovine serum) and cell growth was monitored every $24 \mathrm{~h}$ for 7 days. The next day, the CA IV overexpression cells were incubated with CCK- 8 for $1 \mathrm{~h}$, and the absorbance was measured at $450 \mathrm{~nm}$ using a multifunctional microplate reader (Tecan, Männedorf, Switzerland) in the $1,3,5$, and $7 \mathrm{~d}$. This experiment was done in quadruplicate cells.

Statistical methods. Statistical analysis was performed by SPSS software v19 (SPSS Inc., Chicago, IL, USA). Statistical differences in CA IV mRNA expression levels among different groups were calculated using the non-parametric Kruskal-Wallis H test and Mann-Whitney U test for the skewed distribution or the one-way ANOVA and Dunnett's test for the normal distribution after the Kolmogorov-Smirnov test. The Kaplan-Meier method was used to calculate the overall survival rate, and the prognostic significance was evaluated by the $\log$-rank test. $\mathrm{P}<0.05$ was considered to indicate a statistically significant difference.

\section{Results}

The expression level of CA IV mRNA in NSCLC and adjacent tissues and its relationship with clinical data. Overall, CA IV mRNA expression level of NSCLC is $0.543(0.032-0.956)$ and markedly lower than its adjacent normal tissues (Mann-Whitney $\mathrm{U}=0.000, \mathrm{P}<0.001$ ) (Fig. 1). According to Table I, we observed that the CA IV level of NSCLC with lymph node metastasis group was significantly lower than that of NSCLC without lymph node metastasis group (Mann-Whitney $\mathrm{U}=1.712$, $\mathrm{P}=0.015)$. CA IV expression levels among five TMN stages were not different (Kruskal-Wallis $\mathrm{H}$ test=147.231, $\mathrm{P}=0.575$ ). The CA IV $m R N A$ expression was also not relative to the histology differentiation (Kruskal-Wallis $\mathrm{H}$ test $=5.345$, $\mathrm{P}=0.712$ ), smoking habits (Mann-Whitney $\mathrm{U}=243.12, \mathrm{P}=0.312$ ), gender (Mann-Whitney $\mathrm{U}=235.12, \mathrm{P}=0.643$ ), and histological subtype (Kruskal-Wallis $\mathrm{H}$ test=5.320, $\mathrm{P}=0.724$ ).

The relation of $C A I V m R N A$ expression to the prognosis of NSCLC. The overall survival time of low CA IV expression 
Table I. Relationship between clinical pathological features and CAIV mRNA expression levels in 114 cases of patients with NSCLC.

\begin{tabular}{|c|c|c|c|c|}
\hline Parameter & Cases & $\begin{array}{c}2^{-\Delta \Delta C q} \text { of CA IV mRNA } \\
\text { Median (range) }\end{array}$ & $\begin{array}{l}\text { Kruskal-Wallis H or } \\
\text { Mann-Whitney test }\end{array}$ & P-value \\
\hline Gender & 114 & & 235.12 & 0.643 \\
\hline Male & 56 & $0.445(0.027-0.945)$ & & \\
\hline Female & 58 & $0.556(0.025-0.887)$ & & \\
\hline TMN stage & 114 & & $147.231^{\mathrm{a}}$ & 0.575 \\
\hline Ia & 24 & $0.532(0.554-0.942)$ & & \\
\hline $\mathrm{Ib}$ & 56 & $0.511(0.499-0.856)$ & & \\
\hline IIa & 14 & $0.359(0.215-0.656)$ & & \\
\hline IIb & 4 & $0.224(0.092-0.535)$ & & \\
\hline IIIa & 16 & $0.167(0.077-0.339)$ & & \\
\hline Histological degree & 114 & & $5.345^{\mathrm{a}}$ & 0.712 \\
\hline Poor & 22 & $0.575(0.132-0.945)$ & & \\
\hline Poor-moderate & 14 & $0.414(0.121-0.945)$ & & \\
\hline Moderate & 34 & $0.323(0.082-0.876)$ & & \\
\hline Moderate-high & 18 & $0.323(0.097-0.819)$ & & \\
\hline High & 26 & $0.281(0.095-0.778)$ & & \\
\hline Lymph node metastasis & 114 & & 1.712 & 0.015 \\
\hline Yes & 28 & $0.054(0.025-0.164)$ & & \\
\hline No & 86 & $0.431(0.287-0.943)$ & & \\
\hline Smoking & 114 & & 243.12 & 0.312 \\
\hline Yes & 40 & $0.431(0.026-0.931)$ & & \\
\hline No & 74 & $0.421(0.021-0.923)$ & & \\
\hline histological subtype & & & 5.320 & 0.724 \\
\hline LAD & 68 & $0.412(0.031-0.921)$ & & \\
\hline $\mathrm{SCC}$ & 40 & $0.429(0.026-0.927)$ & & \\
\hline Big cell cancer & 16 & $0.412(0.021-0.908)$ & & \\
\hline
\end{tabular}

LAD, lung Adenocarcinoma; SCC, squamous cell carcinoma. ${ }^{a}$ Kruskal-Wallis H test.

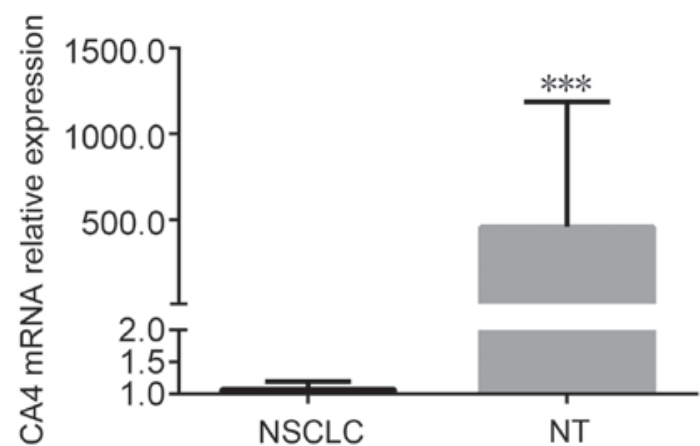

Figure 1. The relative expression levels of CAIV in NSCLC and NT tissues. CAIV expression level of NSCLC is 0.521 (0.025-0.987) and markedly lower than its adjacent normal tissues $(\mathrm{P}<0.001) .{ }^{* * * *} \mathrm{P}<0.001$.

group (median 9 months) was marginally lower than that of the high expression (median 26 months) $\left(\chi^{2}=18.36, \mathrm{P}<0.001\right)$ (Fig. 2). Reduced CA IV expression was associated with shorter overall survival in patients with NSCLC. This finding suggests that reduced CA IV $m R N A$ expression was a predictive factor of poor survival in NSCLC.

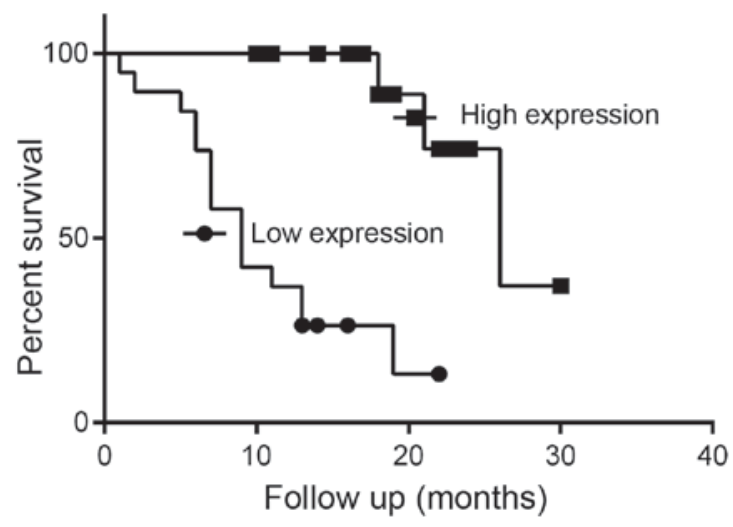

Figure 2. Kaplan-Meier analysis estimates of cumulative overall survival in patients with NSCLC. The overall survival time of low CAIV expression group was marginally lower than that of the high expression $\left(\chi^{2}=18.36\right.$, $\mathrm{P}<0.001)$.

The expression level of CA IV mRNA from six NSCLC cell lines. We detected the expression levels of CA IV from six NSCLC cell lines (including SPCA-1, NCI-H1975, LTEP-a2, 


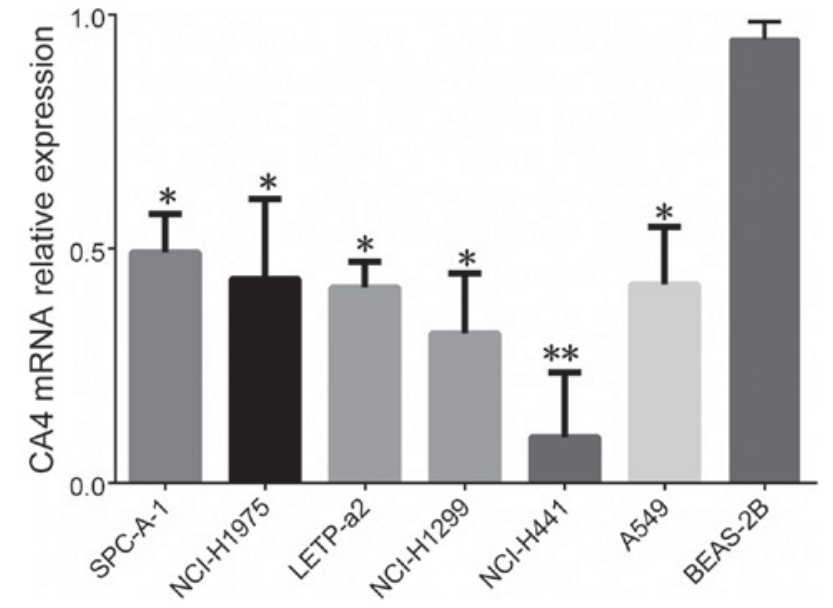

Figure 3. The expression levels of CA IV in six NSCLC cell lines. ${ }^{*} \mathrm{P}<0.05$, ${ }^{* *} \mathrm{P}<0.01$, when compared to BEAS-2B.

NCI-H1299, NCI-H441 and A549) by RT-qPCR. It was shown that the expression levels of CA IV from SPCA-1 $(\mathrm{P}<0.05)$, NCI-H1975 $(\mathrm{P}<0.05)$, LTEP-a2 $(\mathrm{P}<0.05)$, NCI-H1299 $(\mathrm{P}<0.05), \mathrm{A} 549(\mathrm{P}<0.05)$ and NCI-H441 $(\mathrm{P}<0.01)$ were lower, compared to normal human bronchial epithelial BEAS-2B cell line (Fig. 3).

CAIV can regulate ability of cell proliferation. Growth curves of untreatedA549 and NCI-H1299 cells (control), cell stably transduced with non-targeting negative control (NC) and a vector for overexpression of CA IV (CA IV overexpression) were gradually increased with the change of time (Fig. 4A and B). Compared with the $1 \mathrm{~d}$, the OD450 $\mathrm{nm}$ of the 3,5 , and $7 \mathrm{~d}$ in control group were significantly increased $(\mathrm{P}<0.05, \mathrm{P}<0.01$ and $\mathrm{P}<0.001)$, the same results were also found in the NC group and CA IV overexpression group, respectively. Compared with corresponding days of control group or NC group, the OD450 nm of 1, $3 \mathrm{~d}$ in CA IV overexpression group were no statistically significant difference $(\mathrm{P}>0.05)$, while that of the $5 \mathrm{~d}(\mathrm{P}<0.05)$ and the $7 \mathrm{~d}$ $(\mathrm{P}<0.01)$ were significantly reduced, it indicates that cell proliferation ability of A549 and NCI-H1299 lines was significantly suppressed by CA IV overexpression (Fig. 4).

\section{Discussion}

In the present study, we first demonstrated that CA IV mRNA expression was significantly decreased in NSCLC tissues compared with their adjacent normal tissues via RT-qPCR. Compared to normal human bronchial epithelial BEAS-2B cell line, it was shown that the expression levels of CA IV were also reduced in all six NSCLC cell lines. These results suggest an aberrant downregulation of CA IV in NSCLC and hint CA IV gene may be a potent tumor suppressor molecule. Notably, other members of the carbonicanhydrase family were hypoxia-inducible molecules in various types of solid cancers with a more aggressive phenotype and upregulated by hypoxia-inducible factor 1 (HIF-1) (21-23). In order to further study the mechanism of CA IV, we established CA IV overexpression of A549 and NCI-H1299 cell lines by lentivirus-mediated technology. After CA IV was overexpressed, cell proliferation ability of A549 and NCI-H1299 remarkably
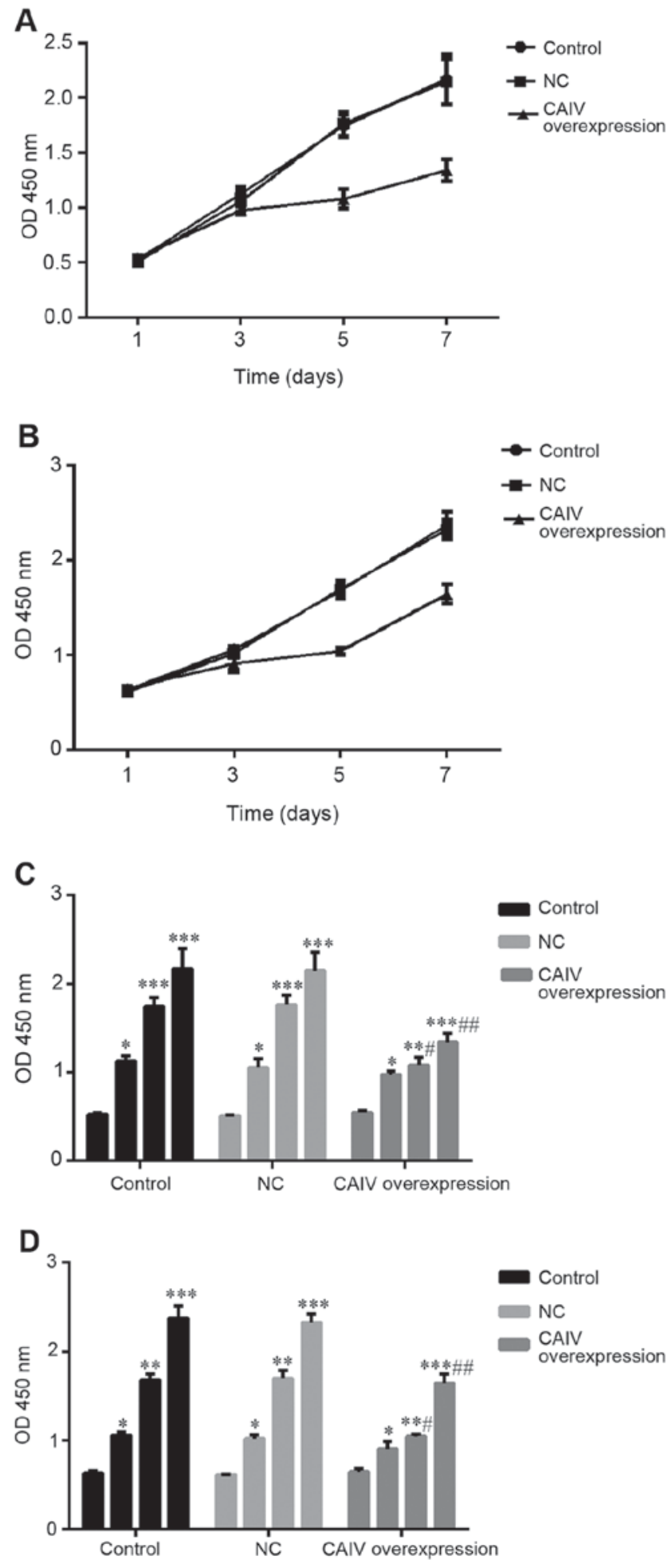

Figure 4. The cell proliferation results of different A549 and NCI-H1299 groups. (A) Growth curves of four days in different A549 groups. (B) Growth curves of four days in different NCI-H1299 groups. Growth curves show that the absorbance of each cell at $450 \mathrm{~nm}$ gradually increased over time, respectively. (C) The line boxplots of different A549 groups at four time points. (D) The line boxplots of different NCI-H1299 groups at four-time points. Compared with corresponding days of the control group or NC group, the OD450 nm of 1d, 3d in CA IV overexpression group were no statistically significant difference, while that of the $5 d(P<0.05)$ and the $7 d(P<0.01)$ significantly reduced. ${ }^{*} \mathrm{P}<0.05,{ }^{* *} \mathrm{P}<0.01,{ }^{* * *} \mathrm{P}<0.001 ;{ }^{\#} \mathrm{P}<0.05,{ }^{\# \#} \mathrm{P}<0.01$.

decreased. Based on these observations, we conclude that low expression of CA IV promotes cell proliferation and CA IV 
acts as a tumor suppressor gene. To the best of our knowledge, this is the first identified that CA IV expression associated with tumor suppressor potential in NSCLC. Zhang shown that CA4 was silenced in all nine colon cancer cell lines and $92.6 \%$ of colon cancer. The re-expression of CA4 inhibited cell proliferation, induced apoptosis and cell cycle arrest in the G1 phase. CA4 inhibited the activity of the Wnt signalling pathway and mediated the degradation of $\beta$-catenin. CA4 interacted with Wilms' tumour 1-associating protein (WTAP) and induced WTAP protein degradation through polyubiquitination. Moreover, CA4 promoted the transcriptional activity of Wilms' tumour 1 (WT1), an antagonist of the Wnt pathway, which resulted in the induction of transducin $\beta$-like protein 1 (TBL1) and the degradation of $\beta$-catenin (18), we will carry out an in-depth study about the mechanism of CA IV in the NSCLC.

The diagnostic and prognostic value of carbonic anhydrases were proved in many solid tumors. It is generally acknowledged that overexpression of CAs can predict poor survival of patients, containing those with NSCLC $(14,24)$. However, clinical properties and prognostic significance of CA IV in NSCLC remain unclear. In our study, the CA IV expression in NSCLC with lymph node metastasis group was significantly lower than that of NSCLC without lymph node metastasis group, while it was not relative to TMN stages, histology differentiation, gender, and smoking. Survival analysis showed that survival time of low expression CA IV group was significantly shorter than high expression CA IV group in NSCLC patients. Low CA IV expression is associated with cell proliferation and lymph node metastasis, which means that low CA IV expression accelerates tumor growth and aggressiveness, resulting in a poor prognosis. Those findings suggest that reduced CA IV expression is a predictive factor of poor survival in NSCLC.

To summarize, our studies ascertain for the first time that the expression of CA IV is downregulated in NSCLC and associated with promoting cell proliferation and lymph node metastasis. The low expression of CA IV may serve as an indicator of poor prognosis in NSCLC.

\section{Acknowledgements}

The present study was financially supported by the National Natural Science Foundation of China (8140736, 81672088), the Zhejiang Provincial Natural Science Foundation (LQ16H160020), and the Wenzhou Municipal Science and Technology Bureau, China (Y20150097).

\section{References}

1. Chen W, Zheng R, Baade PD, Zhang S, Zeng H, Bray F, Jemal A, $\mathrm{Yu}$ XQ and He J: Cancer statistics in China, 2015. CA Cancer J Clin 66: 115-132, 2016.

2. Siegel R, Naishadham D and Jemal A: Cancer statistics, 2013. CA Cancer J Clin 63: 11-30, 2013.

3. Jemal A, Siegel R, Ward E, Hao Y, Xu J, Murray T and Thun MJ: Cancer statistics, 2008. CA Cancer J Clin 58: 71-96, 2008.

4. Stinchcombe TE and Socinski MA: Current treatments for advanced stage non-small cell lung cancer. Proc Am Thorac Soc 6: 233-241, 2009.

5. Mahalingam D, Mita A, Mita MM, Nawrocki ST and Giles FJ: Targeted therapy for advanced non-small cell lung cancers: Historical perspective, current practices, and future development. Curr Probl Cancer 33: 73-111, 2009.
6. Ogawa E, Takenaka K, Katakura H, Adachi M, Otake Y, Toda Y, Kotani H, Manabe T, Wada H and Tanaka F: Perimembrane Aurora-A expression is a significant prognostic factor in correlation with proliferative activity in non-small-cell lung cancer (NSCLC). Ann Surg Oncol 15: 547-554, 2008.

7. Waheed A and Sly WS: Membrane associated carbonic anhydrase IV (CA IV): A personal and historical perspective. Subcell Biochem 75: 157-179, 2014

8. Tashian RE: The carbonic anhydrases: Widening perspectives on their evolution, expression and function. BioEssays: News and reviews in molecular, cellular and developmental biology 10: 186-192, 1989.

9. Zhu XL and Sly WS: Carbonic anhydrase IV from human lung. Purification, characterization and comparison with membrane carbonic anhydrase from human kidney. J Biol Chem 265: 8795-8801, 1990

10. Carter ND, Fryer A, Grant AG, Hume R, Strange RG and Wistrand PJ: Membrane specific carbonic anhydrase (CAIV) expression in human tissues. Biochim Biophys Acta 1026: 113-116, 1990

11. Wang DB, Lu XK, Zhang X, Li ZG and Li CX: Carbonic anhydrase 1 is a promising biomarker for early detection of non-small cell lung cancer. Tumour Biol 37: 553-559, 2016.

12. Ilie M, Hofman V, Zangari J, Chiche J, Mouroux J, Mazure NM, Pouysségur J, Brest P and Hofman P: Response of CAIX and CAXII to in vitro re-oxygenation and clinical significance of the combined expression in NSCLC patients. Lung cancer 82: 16-23, 2013.

13. Malentacchi F, Simi L, Nannelli C, Andreani M, Janni A, Pastorekova S and Orlando C: Alternative splicing variants of carbonic anhydrase IX in human non-small cell lung cancer. Lung cancer 64: 271-276, 2009.

14. Giatromanolaki A, Koukourakis MI, Sivridis E, Pastorek J, Wykoff CC, Gatter KC and Harris AL: Expression of hypoxia-inducible carbonic anhydrase-9 relates to angiogenic pathways and independently to poor outcome in non-small cell lung cancer. Cancer Res 61: 7992-7998, 2001.

15. Kim SJ, Rabbani ZN, Dewhirst MW, Vujaskovic Z, Vollmer RT, Schreiber EG, Oosterwijk E and Kelley MJ: Expression of HIF-1alpha, CA IX, VEGF, and MMP-9 in surgically resected non-small cell lung cancer. Lung cancer 49: 325-335, 2005.

16. Lu SH, Takeuchi T, Fujita J, Ishida T, Akisawa Y, Nishimori I, Kohsaki T, Onishi S, Sonobe H and Ohtsuki Y: Effect of carbonic anhydrase-related protein VIII expression on lung adenocarcinoma cell growth. Lung cancer 44: 273-280, 2004.

17. Wong BC, Zhang H, Qin L, Chen H, Fang C, Lu A and Yang Z: Carbonic anhydrase IX-directed immunoliposomes for targeted drug delivery to human lung cancer cells in vitro. Drug Des Devel Ther 8: 993-1001, 2014.

18. Zhang J, Tsoi H, Li X, Wang H, Gao J, Wang K, Go MY, Ng SC, Chan FK, Sung JJ and Yu J: Carbonic anhydrase IV inhibits colon cancer development by inhibiting the Wnt signalling pathway through targeting the WTAP-WT1-TBL1 axis. Gut 65: 1482-1493, 2016.

19. Brambilla E, Travis WD, Colby TV, Corrin B and Shimosato Y: The new World Health Organization classification of lung tumours. Eur Respir J 18: 1059-1068, 2001.

20. Ren S, Peng Z, Mao JH, Yu Y, Yin C, Gao X, Cui Z, Zhang J, Yi K, $\mathrm{Xu}$ W, et al: RNA-seq analysis of prostate cancer in the Chinese population identifies recurrent gene fusions, cancer-associated long noncoding RNAs and aberrant alternative splicings. Cell Res 22: 806-821, 2012.

21. Malentacchi F, Vinci S, Melina AD, Kuncova J, Villari D, Nesi G, Selli C, Orlando C, Pazzagli M and Pinzani P: Urinary carbonic anhydrase IX splicing messenger RNA variants in urogenital cancers. Urol Oncol 34: 292.e9-292.e16, 2016.

22. Ambrosio MR, Di Serio C, Danza G, Rocca BJ, Ginori A, Prudovsky I, Marchionni N, Del Vecchio MT and Tarantini F: Carbonic anhydrase IX is a marker of hypoxia and correlates with higher Gleason scores and ISUP grading in prostate cancer. Diagn Pathol 11: 45, 2016.

23. Jamali S, Klier M, Ames S, Barros LF, McKenna R, Deitmer JW and Becker HM: Hypoxia-induced carbonic anhydrase IX facilitates lactate flux in human breast cancer cells by non-catalytic function. Sci Rep 5: 13605, 2015.

24. van Kuijk SJ, Yaromina A, Houben R, Niemans R, Lambin P and Dubois LJ: Prognostic significance of carbonic anhydrase IX expression in cancer patients: A meta-analysis. Front Oncol 6: 69, 2016. 